\title{
FREQUENCY-DEPENDENT RECRUITMENT OF INHIBITION MEDIATED BY STELLATE CELL IN THE RAT CEREBELLAR CORTEX
}

\author{
A. RANCILLAC* and J.G. BARBARA* \\ Neurobiologie des Processus Adaptatifs, UMR 7102, Université Pierre et Marie Curie, Paris, France
}

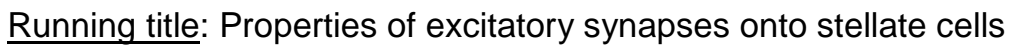

${ }^{*}$ Corresponding author:

Armelle Rancillac

Neurobiologie des Processus Adaptatifs, UMR 7102

UPMC, Case 16

7, Quai Saint Bernard,

75252 Paris Cedex 05, France

Tel : 33 - (0)144272587

Fax : 33 - (0)1 44272584 


\begin{abstract}
In the cerebellum, dendritic inhibition of Purkinje cells (PCs) is mediated by stellate cells (SCs). These inhibitory interneurons are critically involved in the cerebellar network as they control the timing and firing frequency of PCs, which are the only output cells of the cerebellar cortex. However, the underlying properties of parallel fiber (PF) to SC excitatory synapses have not been fully determined. To characterize the conditions favoring the recruitment of dendritic inhibition in the cerebellum, we analyzed evoked and spontaneous EPSCs recorded from SCs of rat cerebellar slices. We found that SC EPSCs evoked with single low intensity stimulations were mostly unitary, with a large amplitude, variable latencies and failed with a high rate (20-40\%). Decreasing failures of PF-SC synapses experimentally moderately increased the mean SC EPSC amplitude, reduced the variability in SC EPSC latency and enhanced the number of asynchronous SC EPSCs per stimulation. Increasing the frequency of stimulations above $60 \mathrm{~Hz}$ significantly reduced failures and moderately synchronized unitary EPSCs. Finally, brief presynaptic bursts transiently depressed synaptic transmission. This depression resulted from the release of endocannabinoids and might act as a negative feedback mechanism. Thus, we concluded that SC EPSCs evoked with single low intensity stimulations are mostly unitary, and that FP-SC synapse efficacy is highly regulated by the presynaptic temporal pattern of activity and the frequency of afferent inputs. Such synaptic properties may control the sensitivity of SC synapses to the frequency of PF stimulations which may control the spatial extent and duration of the recruitment of inhibition in the cerebellar cortex.
\end{abstract}

Key Words : glutamatergic transmission, unitary events, endocannabinoids, asynchronous release, failure rate 
In the cerebellar cortex, stellate cells (SCs) form a dense population of interconnected interneurons that mediate an inhibition from parallel fibres (PF) to Purkinje cell (PC) dendrites (Eccles et al., 1966; Midtgaard, 1992). The spontaneous firing of SCs generates a widespread dendritic GABAergic inhibition in PCs and modulates their firing pattern (Callaway et al., 1995; Häusser and Clark, 1997). In addition, the precise timing of a single SC stimulation can transiently interrupt the firing of a connected PC (Midtgaard, 1992; Häusser and Clark, 1997).

Recently, several studies have concentrated on the functional properties of excitatory synapses between PF and SCs. It was thus established that synaptic transmission is exclusively mediated by AMPA receptors permeant to calcium at low stimulus intensity, whereas extrasynaptic NMDA receptors can be recruited by repetitive or higher intensity stimuli (Carter and Regehr, 2000; Clark and Cull-Candy, 2002). Moreover, synaptic transmission at PF-SC synapses is characterized by a significant amount of release asynchrony (Atluri and Regehr, 1998), even when stimulating PF with stimulus intensities near threshold (Clark and Cull-Candy, 2002). Finally, miniature SC EPSCs suggest that quantal size at PF-SC synapses is high thereby allowing quantal events to shape spontaneous firing of SCs (Carter and Regehr, 2002).

With the exception of a recent study by Clark and Cull-Candy (2002), the influence of the pattern of PF activation on the activity of PF-SC synapses has not yet been considered, despite the fact that this parameter may be important for the mode of operation of synapses (Tsodyks et al., 1998). Therefore, the aim of the present study was to analyze the influence of different patterns of presynaptic activities, ranging from single stimuli to high frequency bursts known to occur in GCs (Eccles et al., 1967), on the function of PF-SC synapses. In keeping with recent observations by Carter and Regehr (2002), we were particularly interested to determine such properties in the case of evoked synaptic responses involving a minimal number of PF-SC synapses.

Here we show that the majority of PF-EPSCs evoked by low intensity PF stimulations are unitary and characterized by a large amplitude, high failure rate, and a large variability in EPSC latency. We also show that while reliability is low for single stimulations, short bursts of presynaptic activity are more efficient. Finally, short-term plasticity depending on release of endocannabinoids can be induced with high frequency 
trains of stimulations. These properties elucidate the synaptic excitation of SCs in relation to the frequency of afferent activity from PF. 


\section{EXPERIMENTAL PROCEDURES}

\section{Slice preparation}

All experiments were carried out on parasagittal or frontal slices $(300 \mu \mathrm{m})$ of rat cerebellar vermis (Sprague-Dawley, 14-20 day old males) and in accordance with international guidelines on the ethical use of animals. The number of rats used was kept to the minimum level necessary for the study and efforts were made to minimize their suffering. After inhalation anesthesia with halothane, rats were decapitated and the cerebellum was rapidly removed and placed in ice-free $\left(0-1^{\circ} \mathrm{C}\right)$ normal saline solution, composed of (in $\mathrm{mM}$ ) : $124 \mathrm{NaCl}, 3 \mathrm{KCl}, 2.5 \mathrm{CaCl}_{2}, 1.15 \mathrm{MgSO}_{4}, 1.15 \mathrm{KH}_{2} \mathrm{PO}_{4}, 24 \mathrm{NaHCO}_{3}, 10$ glucose, $330 \mathrm{mOsm} / \mathrm{l}$, at a final pH of 7.4 when equilibrated with $95 \% \mathrm{O}_{2}-5 \% \mathrm{CO}_{2}$. Slices were cut with a Leica VT1000S vibratome (Leica, Nussloch, Germany) and incubated at room temperature in normal saline for at least 1 hour until use. They were then transferred to a recording chamber on an upright microscope (Zeiss, Paris, France).

\section{Solutions and drugs}

Slices were continuously perfused at a rate of $2 \mathrm{ml} / \mathrm{min}$ with oxygenated saline containing $10 \mu \mathrm{M}$ bicuculline methiodide. Electrode resistances were 4-8 $\mathrm{M} \Omega$ when filled with an internal solution containing (in $\mathrm{mM}$ ) : 140 Kgluconate, $8 \mathrm{NaCl}, 10$ Hepes, $2 \mathrm{Mg}-\mathrm{ATP}, 1 \mathrm{CaCl}_{2}, 10 \mathrm{EGTA}$, pH 7.3, $300 \mathrm{mOsm} / \mathrm{l}$. Unless otherwise indicated this solution was used in all experiments. The cesium based internal solution used in some experiments (see Figure Legends) contained (in mM) : $140 \mathrm{CsMeSO}_{4}, 2 \mathrm{NaCl}, 10 \mathrm{CsHEPES}, 1 \mathrm{QX} 314,5$ TEA, $1 \mathrm{CaCl}_{2}$, $2 \mathrm{Mg}$-ATP, $10 \mathrm{CsEGTA}$, pH 7.3, $300 \mathrm{mOsm} / \mathrm{l}$. The basal internal free [ $\left.\mathrm{Ca}^{2+}\right]$ was $10 \mathrm{nM}$ for both internal solutions. When indicated in the text BAPTA $(30 \mathrm{mM})$ was added in the internal solution. Other drugs were added to normal saline. 6-cyano-7-nitroquinoxaline-2-3-dione (CNQX) and D-2-amino-5-phosphopentanoic acid (D-APV) were purchased from Tocris (Illkirch, France), and 8-cyclopentyl-1,3-dimethylxanthine (CPT) from RBI (St. Quentin Fallavier, France). CGP 56999A was a gift from Novartis Pharma AG (Bäsel, Switzerland) and SR 141716A from Sanofi-Synthélabo Recherche (Montpellier, France). All other drugs were purchased from Sigma Aldrich (St. Quentin Fallavier, France). 


\section{Recording procedures}

Recordings were made from SCs located in the outer third of the molecular layer or from PC somata. Cells were visualized using infrared Nomarski optics and a $40 \mathrm{x}$ water immersion objective. Experiments were carried out at room temperature $\left(25-27^{\circ} \mathrm{C}\right)$ unless otherwise indicated. Interneurons were identified by their discharge of spontaneous action potentials in the cell-attached configuration and by the presence of spontaneous IPSCs in whole-cell records. Synaptic currents were recorded in whole-cell voltage-clamp mode at a holding potential of $-60 \mathrm{mV}$ or in current-clamp mode using an Axopatch 200A amplifier (Axon Instruments, Union City, CA, USA). Access resistances were compensated up to $70 \%$ and data were usually filtered at $2 \mathrm{kHz}$. PF were stimulated using a glass monopolar electrode filled with external solution. While there were no differences in amplitude, kinetics or latency for EPSCs recorded either in frontal or sagittal slices, EPSCs were most successfully elicited in frontal slices using stimulating electrodes placed in the molecular layer $>100 \mu \mathrm{m}$ from recorded cells. Low stimulation intensities were used (see Clark and Cull-Candy, 2002; $<200 \mu \mathrm{s},<25 \mathrm{~V}$ ). EPSCs were evoked during a - $10 \mathrm{mV}$ hyperpolarizing voltage pulse. Current transients resulting from these pulses were used to derive values of access resistance, which were displayed during the experiment. Data were rejected if this parameter changed by more than $20 \%$ during a recording. PF volley was recorded as extracellular field potentials using a glass electrode filled with normal saline and placed in the molecular layer 500-800 $\mu \mathrm{m}$ away from the stimulus site. PF stimulation produced a well-characterized extracellular potential. To prevent contamination by postsynaptic signals, PF volley was recorded in the presence of $20 \mu \mathrm{M}$ CNQX and $100 \mu \mathrm{M}$ D-APV.

\section{Data analysis}

EPSCs initiated in SCs by PF stimuli usually occurred after a long and variable latency and often displayed short $(<10 \mathrm{~ms})$ bursts. Due to the large variability in EPSC latency, the amplitude of SC EPSCs was measured at the peak of the initial EPSC which were detected off-line using a threshold detection method (Acquis1 software, Bio-logic, Grenoble, France). Spontaneous events were detected using DAC2 software (Bio-logic, Grenoble, France). This program enables detection of EPSCs, by moving a window on the derivative current trace, using a threshold for EPSC amplitude adjusted to - $10 \mathrm{pA}$. Signal-to-noise ratios of 
current recordings allowed the detection of all EPSCs that could be seen visually. Data are always given as mean \pm sem. The coefficient of variation of EPSC amplitudes was calculated as the ratio between the standard deviation and mean of EPSC amplitudes. The Student's unpaired t test, Kolmogorov-Smirnov tests, $\chi^{2}$ test and Wilcoxon test were used to compare changes in coefficient of variation, experimental data with theoretical distributions, amplitude distributions and changes in failure rates, respectively. The probability level at which the Null hypothesis was rejected was 0.05 unless otherwise indicated. Comparison of histogram distributions calculated from different numbers of observations were made possible using probability density functions (pdf) obtained by normalization of the histogram area to 1. 


\section{RESULTS}

The aim of this study was to describe specific properties of PF-SC EPSCs (SC EPSCs) involved in the recruitment of SCs from the molecular layer of the cerebellar cortex (Fig. 1A). SC EPSCs were elicited by low intensity PF stimulations (see Methods).

\section{Asynchronous EPSCs at PF-SC synapses using low stimulation intensities}

In addition to the fast synchronous release of neurotransmitter, a period of delayed release is observed at many synapses (Atluri and Regehr, 1998). This delayed or asynchronous release is well established at PF-PC and PF-SC synapses (Atluri and Regehr, 1998). However, since asynchronous release is not observed at PF-PC synapses under low stimulation intensity within a $10 \mathrm{~ms}$ time window following stimulation (Fig. 1C, upper panel), it was evaluated at PF-SC synapses. Under these conditions, SC EPSCs of 50-200 pA occurred in bursts (Fig. 1B upper panel, $25.1 \pm 5.8 \%$ of trials for a given cell, $n=10$ cells). This asynchronous release occurred within a $10 \mathrm{~ms}$ time window and distributions of EPSC latency show a large variability of EPSC latencies (Fig. 1B, lower panel). The mean SC EPSC latency was $2.55 \pm 0.10 \mathrm{~ms}(\mathrm{n}=15$ cells). Asynchronous EPSCs in SCs were not due to multiple firing of afferent fibres since the extracellular PF volley near to recorded SCs was not delayed (Fig. 1C, lower panel) and showed little variability. As temperature may affect patterns of asynchronous EPSCs, experiments were performed at $30-33^{\circ} \mathrm{C}$. However, no noticeable difference in these patterns was observed $(n=4$; data not shown). Our data show that PF-SC synapses are characterized by a fast asynchronous component of EPSCs under conditions where asynchronous PC EPSCs do not occur. Thus, the asynchrony of SC EPSCs seems to represent a specific synaptic property that is not due to low temperature, presynaptic action potential doublets or the intensity of the afferent stimulation. 

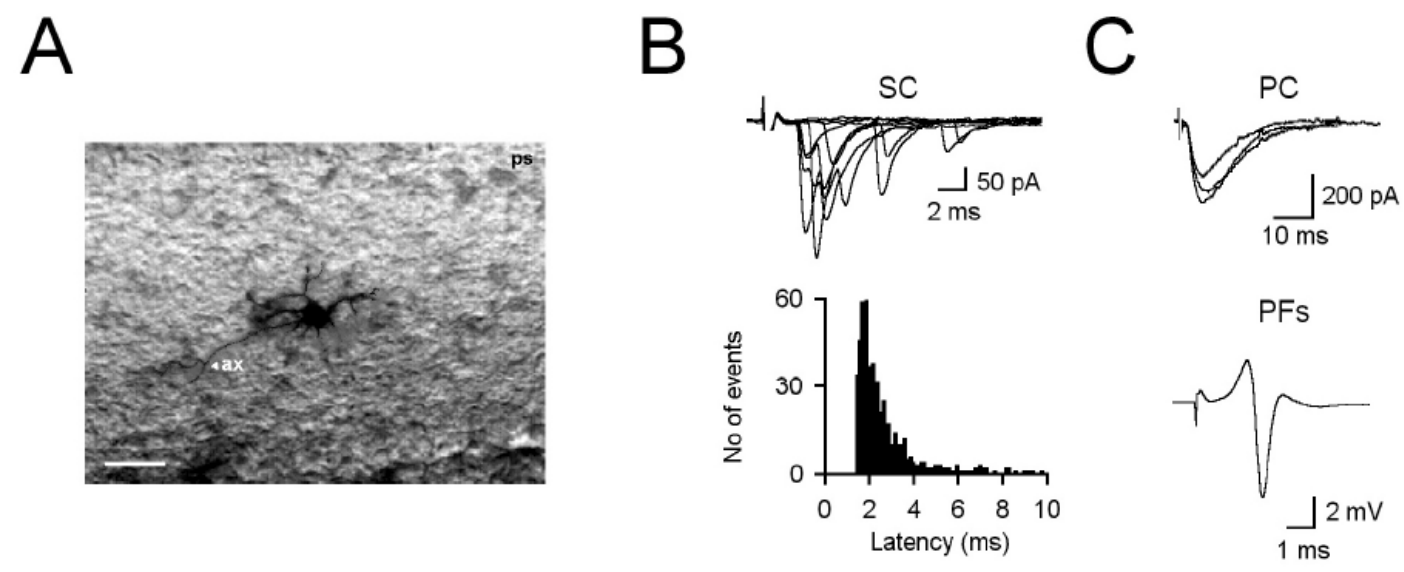

Fig 1. Synaptic properties of the PF-SC synapse. A, Micrograph of a typical SC filled with biocytin in a cerebellar slice. ps, pial surface, ax, axon. Scale bar is $10 \mu \mathrm{m}$. B, Examples of SC EPSCs evoked with a low stimulation intensity showing the variability in latency and asynchronous EPSCs (upper traces). EPSC latency histograms for the SC shown in (B, lower panel). C, Examples of PC EPSCs evoked with the same stimulus intensity as used for SCs (upper traces). Examples of 50 averaged PF volleys (lower traces). For clarity stimulus artefacts are omitted in all current traces.

\section{Are evoked and spontaneous SC EPSCs unitary?}

Synaptic efficacy is in part dependent on the rate of release of neurotransmitter quanta and thus the apparent failure rate. At PF-SC synapses, the asynchrony of SC EPSCs is reminiscent of synapses with a low release probability (Miledi, 1966; Isaacson and Walmsley, 1995; Chuhma and Ohmori, 2002). The apparent failure rate of PF-SC synapses was usually high suggesting that individual SC EPSCs might be unitary. Therefore, this hypothesis was tested on evoked and spontaneous SC EPSCs using strontium $\left(\mathrm{Sr}^{2+}\right)$ which desynchronizes multiquantal EPSCs (Miledi, 1966). 
The unitary nature of SC EPSCs evoked was tested by replacing $\mathrm{Ca}^{2+}$ by $\mathrm{Sr}^{2+}$. $\mathrm{Sr}^{2+}$ reduced the mean EPSC amplitude by $25.5 \pm 3.1 \%$ and no change in the SC EPSC kinetics was observed $(n=10$ cells, Fig. 2A). For comparison, $\mathrm{Sr}^{2+}$ reduced the mean EPSC amplitude by $80.9 \pm 7.2 \%$ in PCs ( $\mathrm{n}=5$ cells, data not illustrated). In the presence of $\mathrm{Sr}^{2+}$, the mean SC EPSC amplitude was $103.5 \pm 14.1 \mathrm{pA}$, with a mean coefficient of variation (see Methods) of $35.5 \pm 3.2 \%$. However, a small increase in the apparent failure rate and a reduction of larger events were observed ( $n=10$ cells, Fig. $2 A$ and B), in agreement with the presence of multiquantal events. We conclude that evoked SC EPSCs are a mixture of unitary and multiquantal events with a majority of unitary events up to 100 pA being observed in most cells.

In order to confirm the large amplitude of unitary EPSCs from a large population of synapses, spontaneous SC EPSCs were examined. While spontaneous EPSCs occurred rarely in SCs (mean frequency $0.26 \pm 0.18$ $\mathrm{Hz}, \mathrm{n}=11$ cells $)$, raising extracellular $\left[\mathrm{K}^{+}\right]$to $20-30 \mathrm{mM}$ increased their frequency to $3.0 \pm 0.6 \mathrm{~Hz}(\mathrm{n}=11$ cells, Fig. 3A and B). These SC EPSCs occurred randomly since intervals between SC EPSCs followed a Poisson distribution (Fig. 3D). Spontaneous SC EPSCs induced with high [K+] had a mean amplitude of 85.7 $\pm 13.5 \mathrm{pA}(\mathrm{n}=11 \mathrm{cells})$ and a mean coefficient of variation of $58.1 \pm 4.8 \%(\mathrm{n}=11$ cells $)$. The unitary nature of spontaneous EPSCs was investigated by comparing cumulative amplitude histograms in control condition and in the presence of $\mathrm{Sr}^{2+}$ or TTX $(1 \mu \mathrm{M})+$ cadmium $\left(\mathrm{Cd}^{2+}, 200 \mu \mathrm{M}\right)$ (Fig. 3C). In 4 out of 4 cells, amplitude distributions were unchanged in the presence of $\mathrm{Sr}^{2+}$ (Kolmogorov-Smirnov tests, $\mathrm{p}>0.2$, Fig. 3E and F). In 7 out of 7 cells, $\mathrm{Cd}^{2+}+$ TTX had no significant effect on amplitude distributions (Kolmogorov-Smirnov test, p>0.2; Fig. 3E and F). When applied alone, TTX $(1 \mu \mathrm{M})$ produced similar results ( $n=3$ cells, data not shown). Finally, the comparison of spontaneous SC EPSC distributions obtained in the presence of TTX $+\mathrm{Cd}^{2+}$ with those of evoked EPSCs revealed no differences (Kolmogorov-Smirnov tests), thereby confirming the unitary nature of the evoked EPSCs. Overall, these data further support that unitary SC EPSCs have a large mean amplitude of 70-100 pA. 


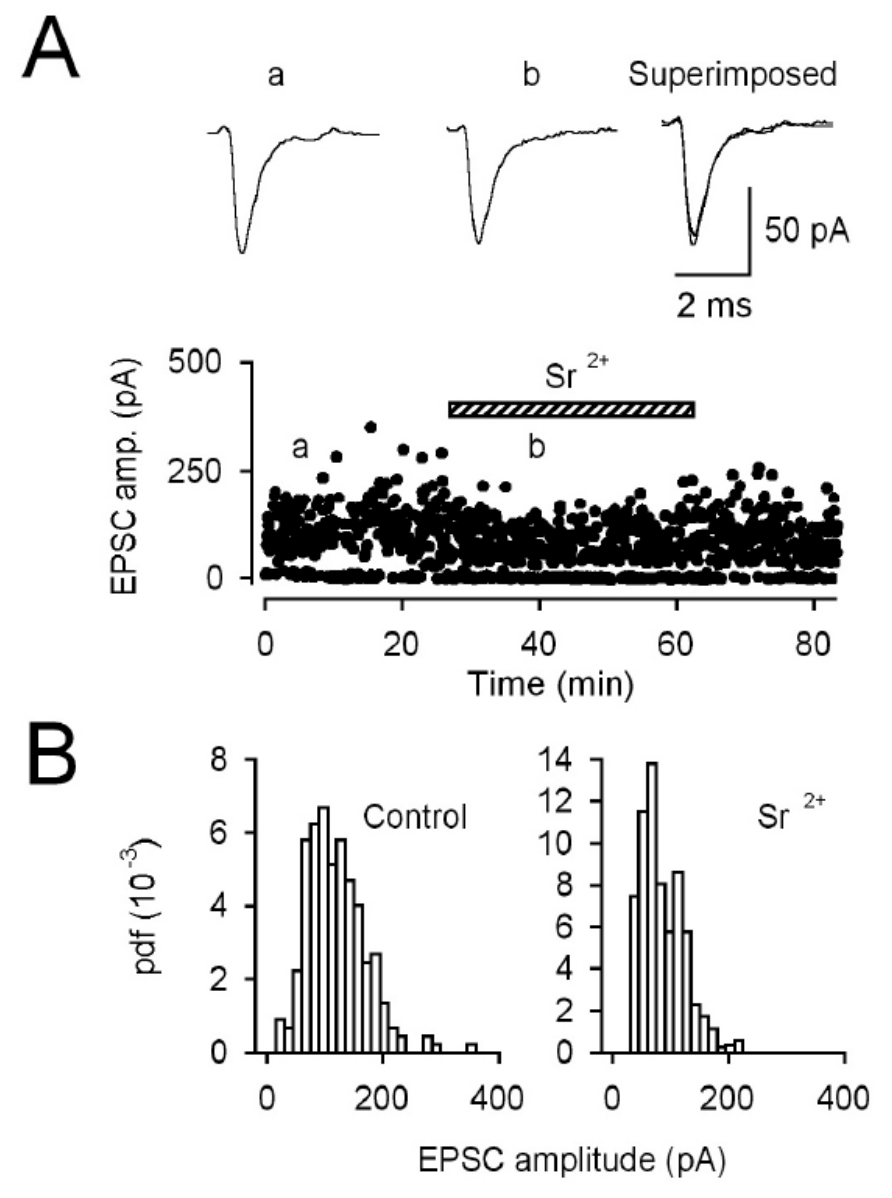

Fig 2. Effect of $\mathrm{Sr}^{2+}$ on evoked SC EPSCs. A, Examples of EPSCs recorded in a typical SC before (a) and after (b) the effect of $\mathrm{Sr}^{2+}$ (top right traces). Superimposed EPSCs of (a) and (b) (top left traces). Plot of the effect of $\mathrm{Sr}^{2+}$ on EPSC amplitude in the same SC. $\mathrm{Sr}^{2+}$ replaced $\mathrm{Ca}^{2+}$ in the bath solution (lower trace). B, Corresponding amplitude histograms showing no effect of $\mathrm{Sr}^{2+}$. pdf : probability density function. 
A

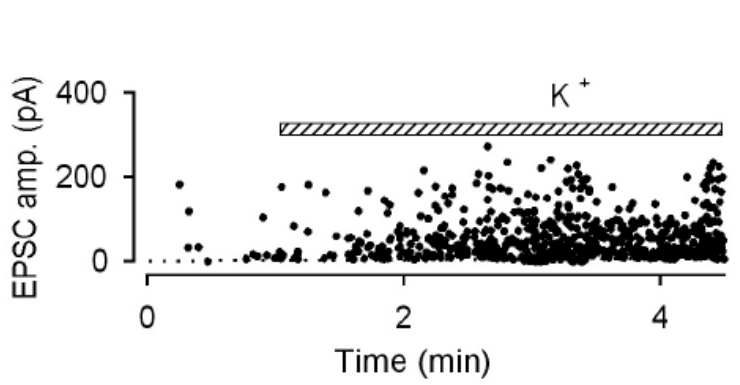

Q
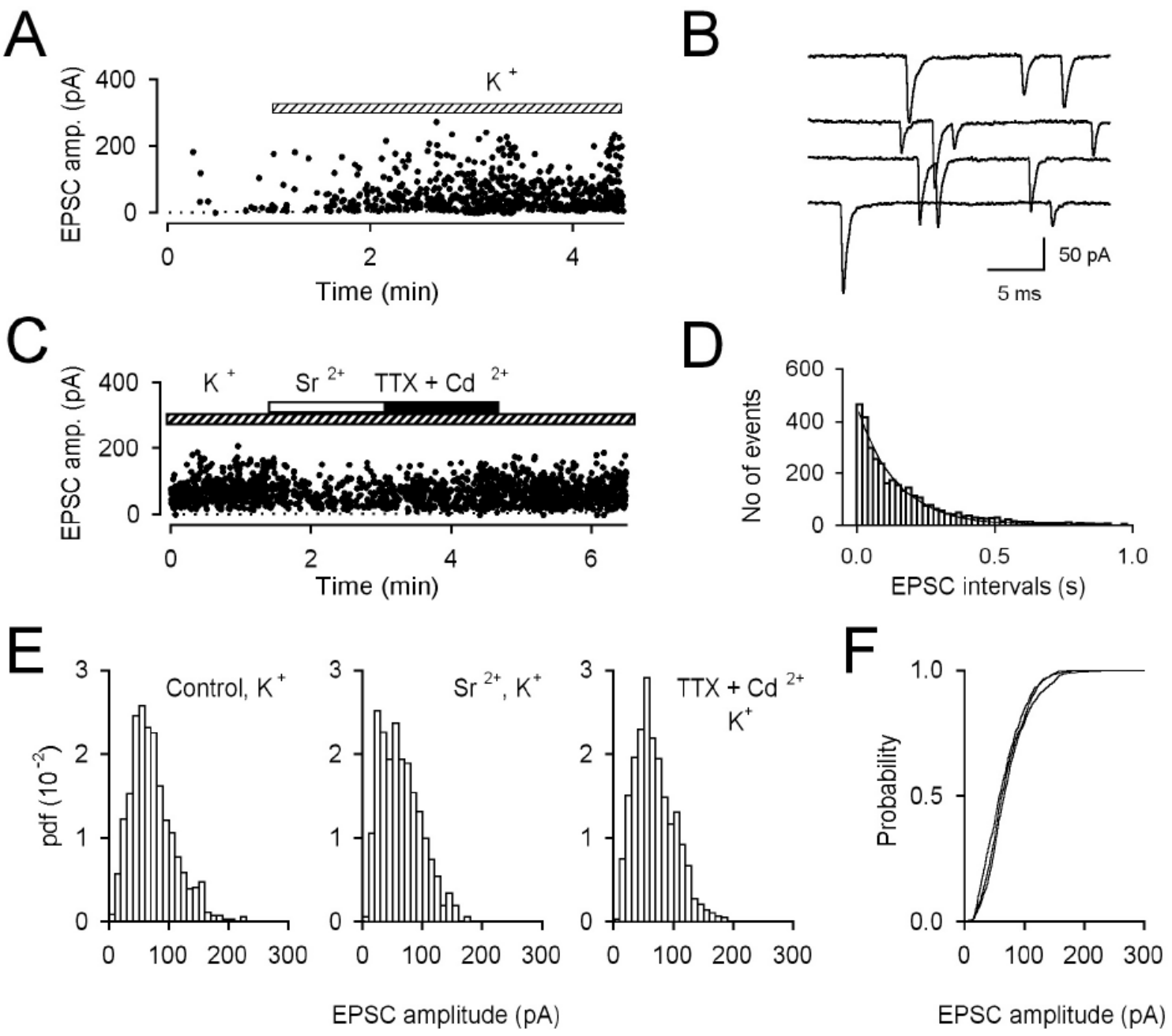

EPSC amplitude (pA)

EPSC amplitude (pA)

Fig 3. $\mathrm{K}^{+}$-induced spontaneous EPSCs are unitary in SCs. A, Application of $\mathrm{KCl}(25 \mathrm{mM})$ dramatically increased spontaneous EPSC frequency in SCs. B, Representative traces of spontaneous SC EPSCs. C, $\mathrm{Sr}^{2+}$ followed by TTX $+\mathrm{Cd}^{2+}$ did not affect $\mathrm{K}^{+}$induced spontaneous SC EPSC amplitude. D, Histogram of SC EPSC intervals fitted with a single exponential. E, SC EPSC amplitude histograms for the previous experimental conditions. F, Histograms from E are cumulated and superimposed for comparison. Cesium based internal solution (see Methods). pdf : probability density function. 


\section{The rate of asynchronous EPSCs at PF-SC synapses is determined by the apparent failure rate}

Altogether, our previous results showed that PF-SC synapses were characterized by a majority of unitary and asynchronous EPSCs. Thus, the efficacy of these synapses does not appear to be determined solely by the amplitude of multiquantal SC EPSCs, but also by the rate of unitary and asynchronous EPSCs. For a given population of synapses, the amplitude of multiquantal EPSCs is dependent on the apparent failure rate. Similarly, the rate of asynchronous SC EPSCs might be correlated with the apparent failure rate at PF-SC synapses. At various synapses, asynchronous EPSCs occur when failures are increased at reduced temperature (Katz and Miledi, 1965), when $\mathrm{Sr}^{2+}$ replaces $\mathrm{Ca}^{2+}$, or when $\mathrm{Ca}^{2+}$ influx is suppressed (Miledi, 1966; Isaacson and Walmsley, 1995, Chuhma and Ohmori, 2002). In these conditions, EPSCs occur asynchronously and their rate conforms to Poisson models (Miledi, 1966; Isaacson and Walmsley, 1995). We thus examined if the rate of asynchronous SC EPSCs was related to the high apparent failure rate observed at PF-SC synapses.

The number of SC EPSCs evoked by each stimulus was counted visually (see Isaacson and Walmsley, 1995). In 8 of 10 cells, the number of failures and the probability that 1, 2, 3, 4 or 5 EPSCs were evoked was well fitted with a Poisson law (no statistical difference between experimental data and theoretical distributions, $\chi^{2}$ test, $p>0.25,80-300$ events per cell, Fig. 4A). The rate of SC EPSCs was correlated to the apparent failure rate (Fig. 4A). Furthermore, the agreement on the number of failures between data and Poisson models suggests that the absence of responses corresponded to true failures of synaptic release and not to failures of stimulation. Altogether, the data show that the number of synchronous events is related to the number of failures at PF-SC synapses. 
A
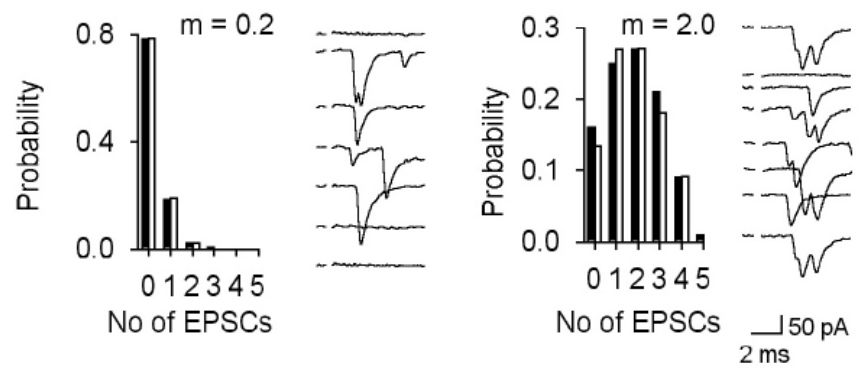

B
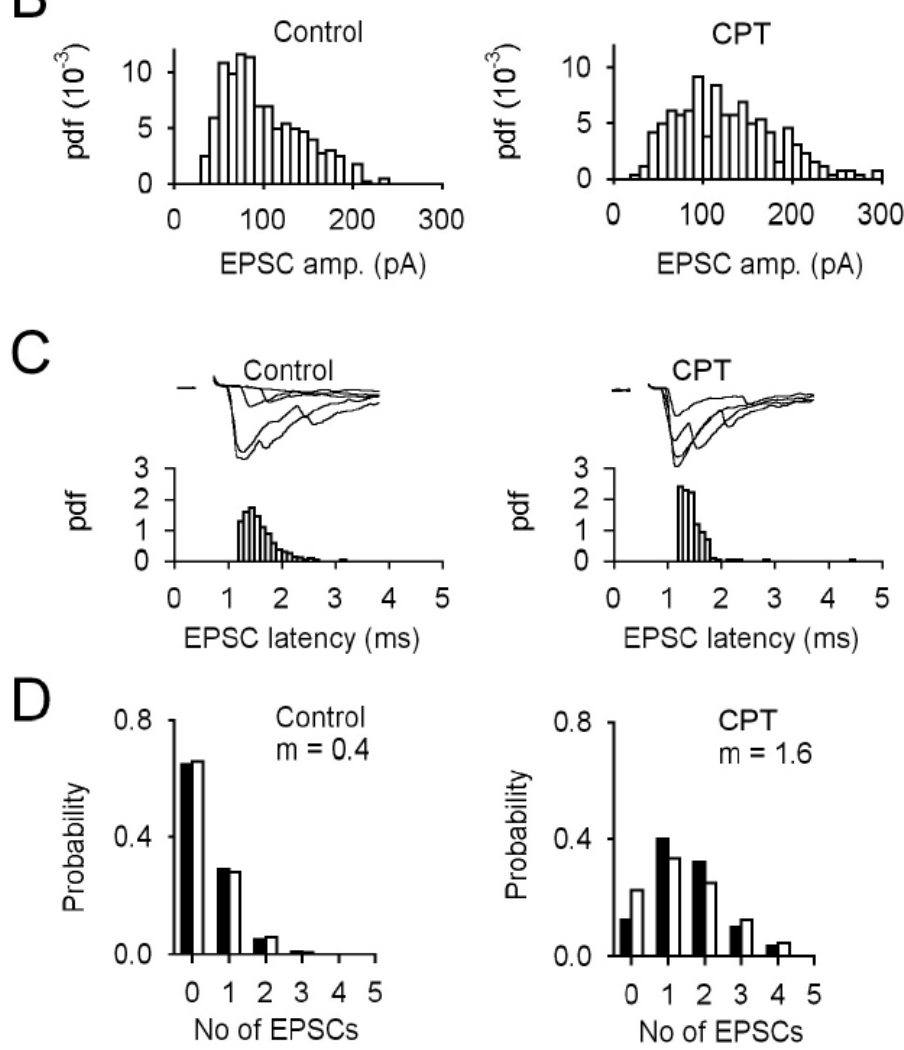

Fig 4. The rate of asynchronous EPSCs per stimulation is dependent on the apparent failure rate. A, Two typical SCs are illustrated. For each cell, example of SC EPSC traces shown with the same scales. The mean number (n) of SC EPSC per stimulation was visually counted. Plots give the observed frequencies of $n$ values (black bars) and the theoretical probabilities of having $0,1, \ldots$ or 5 EPSCs per stimulation calculated with the Poisson law $\left(p(k=x)=m^{x} \cdot e^{-m} / x !\right.$, white bars $)$. B, Failures were decreased using the adenosine antagonist CPT $(1 \mu \mathrm{M})$. The EPSC amplitude histogram is slightly affected by CPT. pdf : probability density function. C, The EPSC latency distribution is narrowed using CPT. D, Effect of CPT on the occurrence of asynchronous EPSCs. 
We next sought to further confirm the relation between asynchronous release in SCs and the apparent failure rate while increasing failures pharmacologically. A first approach was to decrease failures with paired-pulse stimuli. For the second stimulus, the apparent failure rate was reduced by $44.6 \pm 10.1 \%$ (from 0.69 to $0.38, n=10$ cells, data not shown). We also reduced failures using the adenosine receptor antagonist 8-Cyclopentyl-1,3-dimethylxanthine (CPT, $1 \mu \mathrm{M}$, Fig. 4B and C), which caused a decrease in the apparent failure rate by $70.7 \pm 15.4 \%$ (from 0.58 to $0.17, n=9$ cells). Finally, the GABA $B$ antagonist CGP 56999A $(1 \mu \mathrm{M})$ reduced the apparent failure rate by $65.3 \pm 17.0 \%$ (from 0.36 to $0.12, n=9$ cells). EPSC amplitude histograms were moderately affected in each group of experiments (Fig. 4B for CPT) and these changes likely reflected moderate increases in the proportion of multiquantal events. Furthermore, coefficients of variation of EPSC latency distributions were reduced by $29.3 \pm 6.3 \%, 20.4 \pm 2.9 \%$, and 30.1 $\pm 5.9 \%$ respectively for the three treatments (Student's unpaired $t$ test, $p<0.001$, Fig. $4 \mathrm{C}$ for CPT). Conversely, the rate of asynchronous EPSCs was increased and the coefficient of variation of EPSC latency was increased when failures were enhanced by replacing $\mathrm{Ca}^{2+}$ by $\mathrm{Sr}^{2+}$ (data not illustrated). These data further support that decreasing failures pharmacologically has a moderate effect on the appearance of multiquantal events. Thus, the data is not consistent with multiquantal EPSCs being dominant in conditions of low failure rate. The data rather support that asynchronous unitary events are predominant and dependent on the apparent failure rate at PF-SC synapses.

\section{Repetitive stimulations decrease the apparent failure rate of SC EPSCs but do not synchronize them}

Failures at PF-SC synapses were shown to govern the rate of asynchronous unitary SC EPSCs. Since failures are reduced for the second pulse in paired-pulse protocols (Atluri and Regehr, 1998), we tested whether high frequency stimulations (5 stimuli at $100 \mathrm{~Hz}$ ) significantly reduced the apparent failure rate and subsequently increased the efficacy of PF-SC synapses. The high mean apparent failure rate for the first stimulus $(0.86 \pm 0.04, n=7$ cells $)$ decreased regularly for the second, third, fourth and reached a value of $0.34 \pm 0.08$ for the fifth stimulus ( $n=7$ cells, Fig. $5 A$ ). Hence, repetitive stimuli were effective in reducing the apparent failure rate. However, the mean EPSC amplitude for the final EPSC, excluding failures, was only 
increased to $118.3 \pm 15.4 \%$ of that of the first EPSC (Fig. 5A and B). In contrast, similar stimuli trains at PF-PC synapses elicited EPSCs which summed linearly with no apparent failures (data not shown). In agreement with the previous results, these data show that repetitive stimuli strongly decrease failures, but moderately change the proportion of multiquantal EPSCs.

Since high frequency stimulations affected the apparent failure rate at PF-SC synapses, we asked how SC synapses would respond to randomly timed afferent stimuli mimicking short high frequency bursts (100 ms) which they may receive during physiological operation (Eccles et al., 1967). SC EPSCs were recorded over 100 ms periods during which stimulations were applied randomly (Fig. 5C). The distribution of the number (k) of stimulations per 100 ms periods, which we tested, was Gaussian (not shown). The reliability of transmission was quantified using the parameter $\mathrm{N} / \mathrm{k}$, where $\mathrm{N}$ is the number of EPSCs and $\mathrm{k}$ the number of stimulations both per 100 ms periods. We found that $\mathrm{N} / \mathrm{k}$ increased with $\mathrm{k}$ in a remarkably regular manner (Fig. 5D). N/k reached a plateau for $k>6(60 \mathrm{~Hz})$. These data confirm that synaptic failures depend on the frequency of afferent stimuli and suggest that PF-SC synapses function as a high-pass filter of presynaptic activity with transmission becoming reliable above $60 \mathrm{~Hz}$. Thus, the efficacy of PF-SC synapses seems to be governed in part by the apparent failure rate which depends on the frequency of afferent activity. 

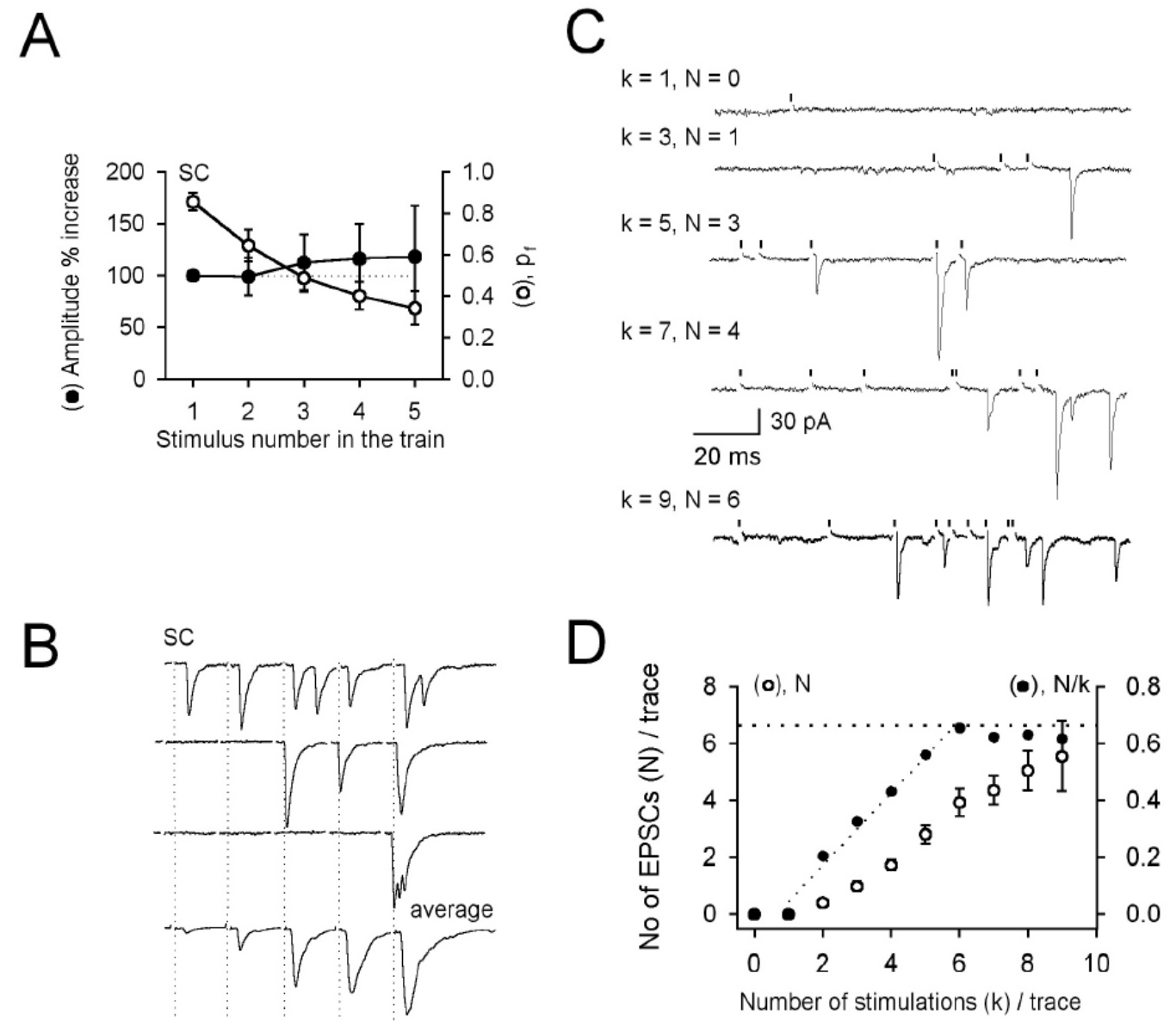

Fig 5. Effect of repetitive random stimulations on synaptic transmission at the PF-SC synapse. A, Plot of the mean EPSC amplitude (closed symbols) and the apparent failure rate ( $\mathrm{p}_{\mathrm{f}}$, open symbols) for each stimulation in a train. The plot is made from 7 cells. Stimulations are separated by $10 \mathrm{~ms}$. The apparent failure rate is reduced within the train while the mean amplitude is moderately increased. B, Representative traces from the same cell (top traces) and 25 averaged SC EPSCs (lower trace). C, Examples of $100 \mathrm{~ms}$ records in which random stimulations were applied. Small vertical bars indicate stimulations ( $k$, number of stimulations and $\mathrm{N}$, number of EPSCs in a $100 \mathrm{~ms}$ record). D, Plot of $\mathrm{N}$ and $\mathrm{N} / \mathrm{k}$ versus $\mathrm{k}$. Note that $\mathrm{N} / \mathrm{k}$ reaches a plateau for $\mathrm{k}>6(60 \mathrm{~Hz})$. 


\section{Multiple high frequency trains transiently depress PF-SC synaptic transmission}

We have shown that PF-SC synapses function more reliably in response to high frequency stimuli. However, repetitive activation might initiate other mechanisms that change synaptic efficacy. In particular, long term changes in the apparent failure rate were observed in the neocortex (Galarreta and Hestrin, 1998). Therefore, the effect of 1 - 4 high frequency trains (100 Hz, $1 \mathrm{~s}, 10 \mathrm{~s}$ interval between trains) was investigated at PF-SC synapses to test for putative mechanisms of synaptic plasticity. Following 4 trains, the apparent failure rate was greatly enhanced and reached a value $>0.5$ within a few minutes $(n=10$ cells, Fig. 6A and $\mathrm{C})$. This depression apparently did not depend on changes in postsynaptic $\mathrm{Ca}^{2+}$. First, maintained somatic depolarizations ( $1-10 \mathrm{~s}$ to $0 \mathrm{mV}$ ) leading to a large $\mathrm{Ca}^{2+}$ influx into cells had no effect and did not occlude the synaptic depression induced by 4 trains ( $n=3$ cells, data not illustrated). Second, BAPTA (30 $\mathrm{mM}$ ) included in the pipette solution did not affect the reduction in synaptic efficacy ( $\mathrm{n}=3$ cells, data not illustrated). These data are in contrast with the effects of repetitive synaptic activity in PCs, which induce a long-term synaptic potentiation with no observed failures (Fig. 6B), as observed previously (Crepel and Jaillard, 1991; Salin et al., 1996). We conclude that high frequency trains produce a strong negative feedback on PF-SC synaptic transmission, which may involve presynaptic mechanisms.

Glutamatergic transmission from PF was shown to be modulated by endocannabinoids released by PCs at the PF-PC synapse (Kreitzer and Regehr, 2001; Brown et al., 2003 ). Potentially, repetitive PF activation could depolarize neighboring PCs, thereby inducing release of endocannabinoids. Consequently, released endocannabinoids may diffuse in the molecular layer and modulate the firing of SCs (Kreitzer et al., 2002; Diana, et al., 2002; Brown et al., 2003). We therefore tested whether the depression of PF-SC transmission is mediated via endocannabinoid signaling. The effect of the type 1 cannabinoid receptor (CB1) antagonist SR 141716A was tested on the transient depression of the PF-SC transmission induced by trains of stimuli. We found that SR $141716 \mathrm{~A}(1 \mu \mathrm{M})$ inhibited the increase in the apparent failure rate observed within 2 min after trains of stimuli (Fig. 6C) and subsequently blocked the depression of PF-SC synaptic transmission. In control conditions, the apparent failure rate was increased from $0.18 \pm 0.06 \%$ to $0.52 \pm 0.10 \%$ after the trains of stimuli (Wilcoxon test, $p<0.01, n=8$ cells). In the presence of SR $141716 \mathrm{~A}$, the apparent failure rate changed from $0.17 \pm 0.04 \%$ to $0.30 \pm 0.06 \%$ ( $p>0.1, n=5$ cells). In these 

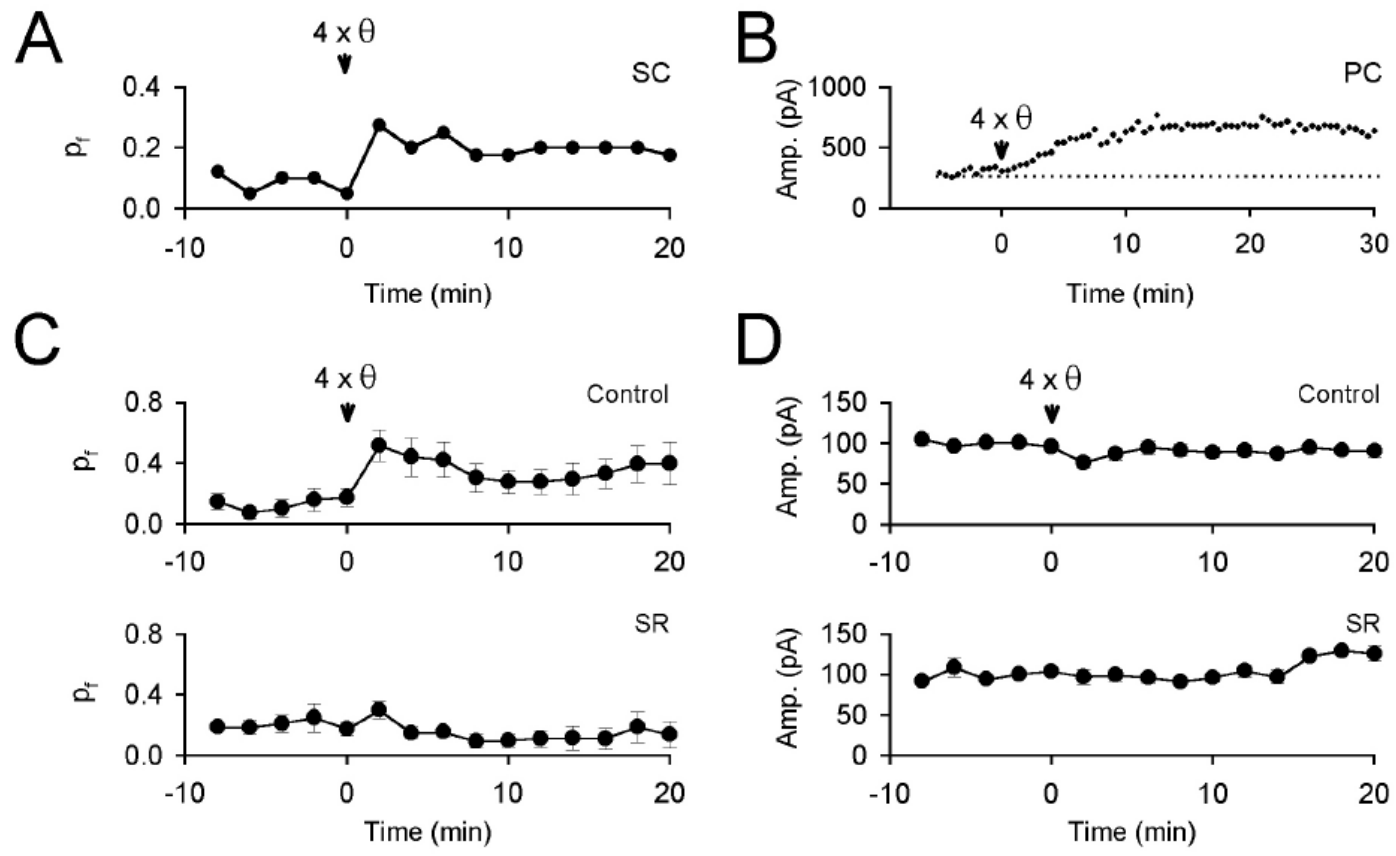

Fig 6 Prolonged and repetitive stimulations transiently depress transmission in SCs. A, Plot of changes in the apparent failure rate over time in a single cell before and after 4 trains of stimulations $(\theta, 100 \mathrm{~Hz}, 1 \mathrm{~s}$, trains separated by $10 \mathrm{~s}) . \mathrm{B}$, In a typical PC, four trains of stimulations induced a longterm potentiation of PC EPSCs. C, Average plot of changes in the mean apparent failure rate over time in response to 4 trains of stimulations (upper plot) : 2 min after stimulations, the apparent failure rate was significantly increased to $294.7 \pm 0.5 \%$ of basal values $(p<0.01, n=8$ cells). In the presence of the CB1 receptor antagonist, SR 141716A $(1 \mu \mathrm{M})$, the apparent failure rate was not significantly changed after stimulations ( $p>0.1, n=5$ cells, lower plot). D, Plots of changes in mean EPSC amplitude following trains of stimulations in control conditions (upper plot) and in the presence of SR 141716A (lower plot). Mean EPSC amplitudes were calculated without taking failures into account. A reduction to $76.1 \pm 6.7 \%$ of basal amplitude was observed in control conditions ( $p<0.02, n=5-8$ cells). No changes were seen in the presence of SR 141716A.

experiments, the mean amplitude of SC EPSCs was reduced to $76.1 \pm 6.7 \% 2 \mathrm{~min}$ after the tetani in control 
conditions ( $p<0.02, n=8$ cells, Fig. $6 D$, upper panel). In agreement with previous data showing a relation between the apparent failure rate and mean EPSC amplitude, these changes are likely to reflect a decrease in the moderate proportion of multiquantal events following the increase in the apparent failure rate induced by tetani. Accordingly, both the apparent failure rate and mean EPSC amplitude were unchanged in the presence of SR 141716A 2 min after the trains of stimuli $(97.2 \pm 9.2 \%, p=0.06, n=8$ cells, Fig. $6 \mathrm{D}$, lower panel). The effects of SR $141716 \mathrm{~A}$ indicate that endocannabinoid signaling may be involved in the presynaptic modulation of release probability at PF-SC synapses. Thus, trains of stimuli transiently depress PF-SC transmission by increasing the apparent failure rate by a mechanism involving the release of endocannabinoids.

\section{Potential impact of PF-SC synapse properties on SC EPSPs}

PF-SC synapse properties were derived from the study of SC EPSCs. However, excitatory postsynaptic potentials (EPSPs) represent the physiologically relevant parameter of synaptic transmission that is integrated temporally and spatially in SCs. Thus, it is important to test the impact of PF-SC synapse properties on SC EPSPs. In particular, the high input resistance of SCs (2-7 G , Llano and Gerschenfeld, 1993) and consequently their high membrane time constants lead to prolonged SC EPSPs (Fricker and Miles, 2000) that might obscure their asynchrony. SC membrane time constants were evaluated from capacitative currents obtained during a - $10 \mathrm{mV}$ hyperpolarization and fitted with two exponentials. Two time constants were derived ( $\tau_{1}=0.17 \pm 0.02 \mathrm{~ms}, \tau_{2}=1.98 \pm 0.40 \mathrm{~ms}, \mathrm{n}=6$ cells $)$. Consequently, the filtering of EPSPs may be significant in SCs and obscure the asynchrony of release. However, individual EPSPs could be resolved within bursts in a time window of $10 \mathrm{~ms}$ after stimulation (Fig. 7A). Therefore, EPSPs closely spaced in a burst could be examined to evaluate the asynchrony of release on SC EPSPs. The number of EPSCs detected per stimulus was often higher in the same cell than the number of detected EPSPs. To determine the time range in which the filtering was significant and might obscure the asynchrony of release, we calculated the distributions of intervals between two closely spaced events for a given cell in voltage-clamp and in current-clamp modes (Fig. 7B). Comparing these distributions revealed that while two EPSCs spaced by $0.2 \mathrm{~ms}$ could be distinguished, a separation of $1 \mathrm{~ms}$ was required to distinguish two EPSPs. Thus, a significant filtering occurred for events spaced by less than $1 \mathrm{~ms}$ and obscured asynchrony. 
Altogether, these data show that although some filtering occurs in a $1 \mathrm{~ms}$ time window, bursts of asynchronous EPSCs at intervals greater than $1 \mathrm{~ms}$ will shape the depolarizing envelope of changes in SC membrane potential.

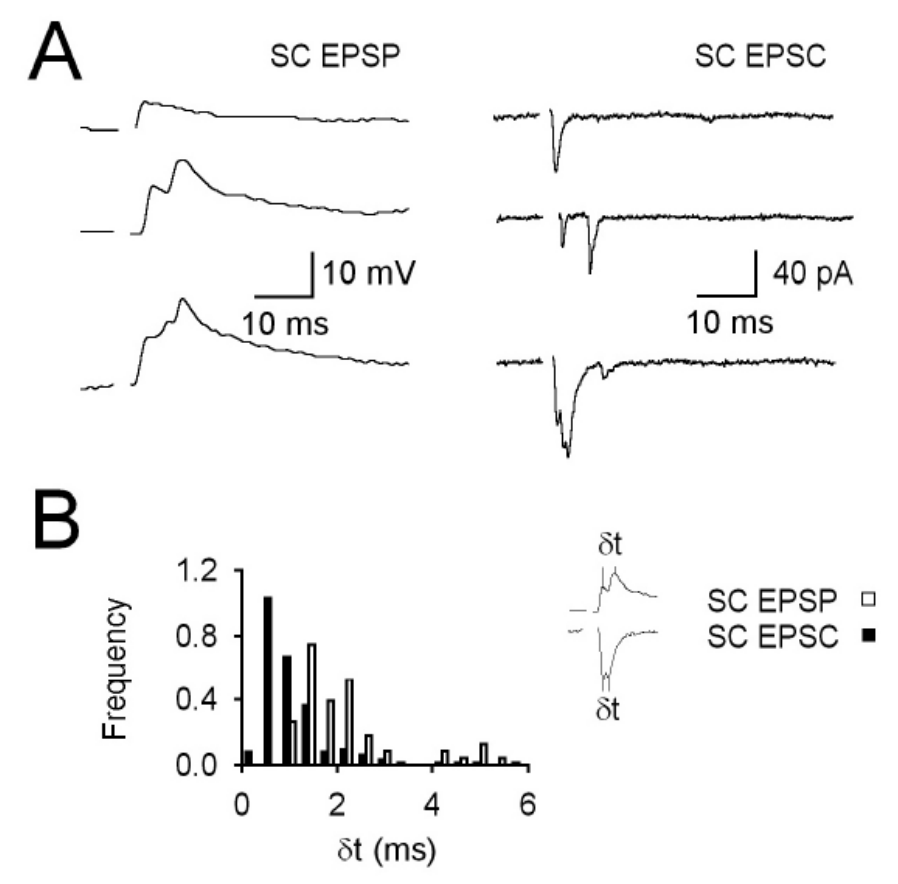

Fig 7. Properties of the PF-SC synapse shape SC EPSPs. A, Voltage-clamp (left traces) and currentclamp recordings (right traces) of SC EPSCs and SC EPSPs from a single SC. Traces were selected to compare the kinetics of individual events or small bursts of SC EPSCs and SC EPSPs respectively.

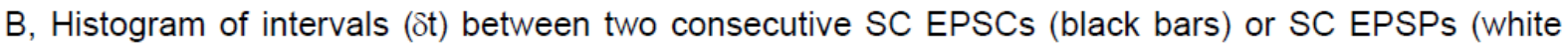
bars) showing that consecutive SC EPSPs can be distinguished when separated by at least $1 \mathrm{~ms}$. SC EPSCs and SC EPSPs were recorded from the same cell. 


\section{DISCUSSION}

We have shown that evoked SC EPSCs are mostly unitary with a significant proportion of multiquantal events and are characterized by their asynchrony and their high apparent failure rate. Although unitary SC EPSCs are sufficient to evoke action potentials in SCs (Barbour, 1993; Carter and Regehr, 2002), the high apparent failure rate and asynchrony of SC EPSCs are limiting factors in the efficacy of these synapses. Decreasing failures by blocking the effect of neuromodulators or with repeated stimulations increased the number of asynchronous SC EPSCs. However, SC EPSCs were only moderately synchronized. Synaptic efficacy at PF-SC synapses is in part governed by the rate of asynchronous SC EPSCs, the frequency of afferent stimulation rather than solely by the amplitude of multiquantal EPSCs and postsynaptic factors. The apparent failure rate was minimal when afferent activity reached $60 \mathrm{~Hz}$. However, at $100 \mathrm{~Hz}$, prolonged synaptic stimulations had a depressant effect on synaptic transmission and this depression was shown to involve the release of endocannabinoids and the activation of CB1 receptors. Thus, PF-SC synapses are highly sensitive to the frequency of afferent PF activity which in turn exerts control over the recruitment of inhibition mediated by SCs in the cerebellar cortex.

\section{Amplitude of unitary SC EPSCs}

The large amplitude of unitary SC EPSCs was in the range of $100 \mathrm{pA}$, in agreement with the assumption of Carter and Regehr (2002) based on measurements of miniature SC EPSCs. A possible explanation for such a large amplitude could be that the number of postsynaptic glutamate receptors may be higher at PF-SC synapses compared to PF-PC synapses. At inhibitory synapses, a correlation was found between synaptic currents and the number of postsynaptic $\mathrm{GABA}_{\mathrm{A}}$ receptors (Nusser et al., 1997). Because the morphology of PF-PC synapses is comparable to that of PF-SC synapses as observed by electron microscopy (Palay and Chan-Palay, 1974), the density of glutamate receptors rather than the surface of the postsynaptic density may be higher at PF-SC synapses.

Large amplitude unitary events are also observed at other synapses including excitatory synapses onto cerebellar Golgi cells (Dieudonné, 1998), glutamatergic synapses of the neonatal hippocampus CA1 region (Hanse and Gustafsson, 2001) and inhibitory synapses onto cerebellar interneurons (Kondo and 
Marty, 1998). At PF-SC synapses, the large amplitude of unitary EPSCs may be functionally related to the poor synchronization of EPSCs which does not allow a build up of EPSCs and to the fact that a single unitary EPSC can produce a suprathreshold depolarization in SCs (Carter and Regehr, 2002).

\section{Asynchronous release at PF-SC synapses}

Our results show that SC EPSCs occur with long, variable latencies and are often followed by asynchronous EPSCs. Asynchronous release at PF-SC synapses was previously described by Atluri and Regehr (1998) mostly at room temperature and with large amplitude EPSCs over $1 \mathrm{nA}$. A fast asynchronous component was shown to last $~ 10 \mathrm{~ms}$ and was calcium-dependent. In our study, a single asynchronous component lasting less than $10 \mathrm{~ms}$ was observed, possibly because low stimulation intensities were used, confirming asynchrony as an intrinsic synaptic property of PF-SC synapses. We showed that the number of asynchronous EPSCs was governed by the apparent failure rate at PF-SC synapses. This asynchronous release is therefore reminiscent of the desynchronized release occurring at other synapses when failures are increased experimentally. In agreement, our data demonstrated a relation between the distribution of EPSC latency and the apparent failure rate. Asynchronous release thus seems to depend on the apparent failure rate and a large variability in EPSC latency under normal conditions at PF-SC synapses. Recent studies have also pointed out similar relations between failures and the EPSC latency during short-term plasticity (reviewed in Lin and Faber, 2002). This general mechanism of plasticity, especially evident at PF-SC synapses, deserves further analysis.

\section{Apparent failures and reliability of PF-SC synapses}

Reliability and plasticity were shown to be sensitive to the frequency of PF activity. Thus, frequency-dependent reliability in SCs allows excitatory synapses to function as frequency detectors. In addition, transmitter spill-over and activation of NMDA receptors are both observed during high frequency activation of these connections with SCs (Carter and Regehr, 2000; Clark and Cull-Candy, 2002). In both cases, five stimulations at $100 \mathrm{~Hz}$ were effective in increasing reliability, as shown in the present study for changes in the apparent failure rate for the fifth stimulus. Therefore, the frequency of afferent activity is an important parameter at PF-SC synapses. This synapse was shown to be reliable at frequencies above 60 
$\mathrm{Hz}$, consistent with patterns of GC activity in vivo which can involve short high frequency bursts (Eccles et al., 1967). Thus, SC synapses generate different temporal patterns of postsynaptic responses, which depend directly on the frequency of PF stimulations.

\section{Transient depression of PF-SC transmission by trains of stimulations}

We found that increasing the stimulation frequency to $100 \mathrm{~Hz}$ during prolonged periods $(4 \times 1 \mathrm{~s}$ trains) transiently depressed transmission at PF-SC synapses. Following trains of stimulations, the apparent failure rate was significantly enhanced and the mean EPSC amplitude was transiently reduced. This phenomenon was independent of postsynaptic calcium suggesting a presynaptic mechanism. A similar frequency-dependent depression has been described in the neocortex (Galarreta and Hestrin, 1998), and we show here that at PF-SC synapses this phenomenon is mediated by the release of endocannabinoids and the activation of $\mathrm{CB} 1$ receptors. The CB1 antagonist SR 141716A blocked both changes in the apparent failure rate and the reduction in the mean EPSC amplitude induced by repetitive stimuli. The change in the apparent failure rate is likely to be mediated by presynaptic CB1 receptors located on PFs (Moldrich and Wenger, 2000). In contrast, the effect on mean EPSC amplitude may result from a reduction of multiquantal events depending on the change in the apparent failure rate, or alternatively may be due to a postsynaptic effect which could involve CB1 receptors located on SCs.

Since the synaptic depression was not blocked by chelating calcium inside SCs, it seems probable that endocannabinoids were released from nearby PCs (see ref. in Kreitzer et al., 2002) or by a calcium-independent mechanism (Maejima et al., 2001) possibly present in SCs. This suggests that endocannabinoids released from PCs following high frequency PF activation might depress not only synaptic transmission to PCs (Kreitzer and Regehr, 2001; Brown et al., 2003) but also that to local SCs. This appears to be the first report of an effect of endocannabinoids released by repetitive afferent activation.

\section{Functional significance of PF-SC excitation for the recruitment of inhibition in the cerebellum}

SCs generally fire at high frequencies $(>10 \mathrm{~Hz})$ in vivo and in vitro at near physiological temperatures (Llano and Gerschenfeld, 1993; Hausser and Clark, 1997). Does this activity arise from spontaneously active 
synaptic excitatory inputs ? In vitro experiments rather suggest that basal glutamatergic synaptic transmission does not significantly contribute to spontaneous SC activity ( Hausser and Clark, 1997; Cohen and Yarom, 1998). This agrees with our observation that spontaneous EPSCs occurred very rarely in SCs recorded in cerebellar slices and also with the low level of spontaneous activity of GCs in cerebellar slices (D'Angelo et al., 1995). Consequently, PF activity has little influence on tonic inhibition from SCs in the cerebellum. Recent data suggest that this type of inhibition probably depends on local circuits made by gap-junctions between SCs (Mann-Metzer and Yarom, 2000). In contrast, massive recruitment of SCs depends on GC activity. Our experiments explain why short episodes of high frequency PF activity recruit SC inhibition more efficiently. Similarly, such activity was shown to synchronize Golgi cells (Vos et al., 1999), resulting in a long-range inhibition (Cohen and Yarom, 2000). It thus seems possible that trains of PF activity will also efficiently synchronize SCs. Because SCs represent the largest population of inhibitory interneurons in the cerebellar cortex, such a synchronization may exert a powerful and widespread inhibition of PCs. Thus, the synaptic sensitivity of SC synapses to the frequency of PF stimulations seems likely to control the spatial extent and duration of the recruitment of inhibition in the cerebellar cortex.

\section{Acknowledgements}

This work was supported by the Centre National de la Recherche Scientifique and Sanofi-Synthélabo Recherche. We thank Jean-Christophe Poncer, Pascal Legendre, Carole Levenes and Isabelle Ferezou for helpful comments, Elisa Hill and Richard Miles for careful reading of the manuscript, Nathalie Leresche for kindly providing CGP 56999A, Isabelle Ferezou and Elisa Hill for help with histochemistry, Daniel Brusciano for expert technical assistance and Gérard Sadoc for Acquis 1 and DAC2 software. 


\section{References}

Atluri PP, Regehr WG (1998) Delayed release of neurotransmitter from cerebellar granule cells. J Neurosci 18:8214-8227.

Barbour B (1993) Synaptic currents evoked in Purkinje cells by stimulating individual granule cells. Neuron 11:759-769.

Brown SP, Brenowitz SD, Regehr WG (2003) Brief presynaptic bursts evoke synapse-specific retrograde inhibition mediated by endogenous cannabinoids. Nat Neurosci 6:1048-1057.

Callaway JC, Lasser-Ross N, Ross WN (1995) IPSPs strongly inhibit climbing fiber-activated $\left[\mathrm{Ca}^{2+}\right]_{\mathrm{i}}$ increases in the dendrites of cerebellar Purkinje neurons. J Neurosci 15:2777-2787.

Carter AG, Regehr WG (2000) Prolonged synaptic currents and glutamate spillover at the parallel fiber to stellate cell synapse. J Neurosci 20:4423-4434.

Carter AG, Regehr WG (2002) Quantal events shape cerebellar interneuron firing. Nat Neurosci $5: 1309-1318$

Chuhma N, Ohmori $\mathrm{H}$ (2002) Role of $\mathrm{Ca}^{2+}$ in the synchronization of transmitter release at calyceal synapses in the auditory system of rat. J Neurophysiol 87:222-228.

Clark BA, Cull-Candy SG (2002) Activity-dependent recruitment of extrasynaptic NMDA receptor activation at an AMPA receptor-only synapse. J Neurosci 22:4428-4436.

Cohen D, Yarom Y (1998) Patches of synchronized activity in the cerebellar cortex evoked by mossy-fiber stimulation : questioning the role of parallel fibers. Proc Natl Acad Sci USA 95:15032-15036.

Cohen D, Yarom Y (2000) Cerebellar on-beam and lateral inhibition : two functionally distinct circuits. J Neurophysiol 83:1932-1940.

Crepel F, Jaillard D (1991) Pairing of pre- and postsynaptic activities in cerebellar Purkinje cells induces long-term changes in synaptic efficacy in vitro. J Physiol 432:123-141.

D'Angelo E, De Filippi G, Rossi P, Taglietti V (1995) Synaptic excitation of individual rat cerebellar granule cells in situ : evidence for the role of NMDA receptors. J Physiol 484:397-413.

Diana MA, Levenes C, Mackie K, Marty A (2002) Short-term retrograde inhibition of GABAergic synaptic currents in rat Purkinje cells is mediated by endogenous cannabinoids. J Neurosci 22:200-208. 
Dieudonné S. (1998) Submillisecond kinetics and low efficacy of parallel fibre-Golgi cell synaptic currents in the rat cerebellum. J Physiol 510:845-866.

Eccles JC, Llinas R, Sasaki K (1966) The inhibitory interneurones within the cerebellar cortex. Exp Brain Res 1:1-16.

Eccles JC, Ito M, Szenthagothai J (1967) The cerebellum as a neuronal machine. Springer Heidelberg.

Fricker D, Miles R (2000) EPSP amplification and the precision of spike timing in hippocampal neurons. Neuron 28:559-569.

Galarreta M, Hestrin S (1998) Frequency-dependent synaptic depression and the balance of excitation and inhibition in the neocortex. Nat Neurosci 1:587-594.

Hanse E, Gustafsson B (2001) Quantal variability at glutamatergic synapses in area CA1 of the rat neonatal hippocampus. J Physiol 531:467-480.

Häusser M, Clark BA (1997) Tonic synaptic inhibition modulates neuronal output pattern and spatiotemporal synaptic integration. Neuron, 19:665-678.

Isaacson JS, Walmsley B (1995) Counting quanta : direct measurements of transmitter release at a central synapse. Neuron 15:875-884.

Katz B, Miledi R (1965) The effect of temperature on the synaptic delay at the neuromuscular junction. J Physiol 181:656-670.

Kondo S, Marty A (1998) Synaptic currents at individual synapses among stellate cells in rat cerebellar slices. J Physiol 509:221-232.

Kreitzer AC, Regehr WG (2001) Retrograde inhibition of presynaptic calcium influx by endogenous cannabinoids at excitatory synapses onto Purkinje cells. Neuron 29:717-727.

Kreitzer AC, Carter AG, Regehr WG (2002) Inhibition of interneuron firing extends the spread of endocannabinoid signaling in the cerebellum. Neuron 34:787-796.

Lin J, Faber D (2002) Modulation of synaptic delay during synaptic plasticity. Trend Neurosci 25:449-455.

Llano I, Gerschenfeld HM (1993) Inhibitory synaptic currents in stellate cells of rat cerebellar slices. J Physiol 468:177-200.

Maejima T, Hashimoto K, Yoshida T, Aiba A, Kano M (2001) Presynaptic inhibition caused by retrograde signal from metabotropic glutamate to cannabinoid receptors. Neuron 31:463-475. 
Mann-Metzer P, Yarom Y (2000) Electrotonic coupling synchronizes interneuron activity in the cerebellar cortex. Prog Brain Res 124:115-122.

Midtgaard J (1992) Stellate cell inhibition of Purkinje cells in the turtle cerebellum in vitro. J Physiol 457:355-367.

Miledi R (1966) Strontium as a substitute for calcium in the process of transmitter release at the neuromuscular junction. Nature 212:1233-1234.

Moldrich G, Wenger T (2000) Localization of the CB1 cannabinoid receptor in the rat brain. An immunohistochemical study. Peptides 21:1735-1742.

Nusser Z, Cull-Candy S, Farrant M (1997) Differences in synaptic GABA(A) receptor number underlie variation in GABA mini amplitude. Neuron 19:697-709.

Palay SL, Chan-Palay V (1974) Cerebellar Cortex : Cytology and organization. Springer Berlin.

Salin PA, Malenka RC, Nicoll RA (1996) Cyclic AMP mediates a presynaptic form of LTP at cerebellar parallel fiber synapses. Neuron 16:797-803.

Tsodyks M, Pawelzik K, Markram H (1998) Neural networks with dynamic synapses. Neural Comput 10:821-835.

Vos BP, Maex R, Volny-Luraghi A, De Schutter E (1999) Parallel fibers synchronize spontaneous activity in cerebellar Golgi cells. J Neurosci 19:RC6 1-15. 